\title{
Comparison of Radiological and Histological Findings of Lung Parenchyma in Patients with Antineutrophil Cytoplasmic Antibody-Associated Vasculitis
}

\author{
Hee-Jin Park ${ }^{1}$, Seung Min Jung ${ }^{2}$, Jason Jungsik Song ${ }^{2}$, Yong-Beom Park ${ }^{2}$, Ji-Sun Song ${ }^{3}$, and Sang-Won Lee ${ }^{2}$ \\ ${ }^{1}$ Division of Rheumatology, Department of Internal Medicine, Catholic Kwandong University College of Medicine, \\ International St. Mary's Hospital, Incheon; \\ ${ }^{2}$ Division of Rheumatology, Department of Internal Medicine, Yonsei University College of Medicine, Seoul; \\ ${ }^{3}$ Department of Pathology, Catholic Kwandong University College of Medicine, International St. Mary's Hospital, Incheon, Korea.
}

\begin{abstract}
Purpose: The present study investigated chest computed tomography (CT) patterns and lung histological features, as well as the consistency between radiological and histological features among patients with microscopic polyangiitis (MPA), granulomatosis with polyangiitis (GPA), and eosinophilic GPA (EGPA).

Materials and Methods: The medical records of 74 antineutrophil cytoplasmic antibody-associated vasculitis (AAV) patients with radiological lung parenchymal lesions were reviewed along with the histological results for 28 of them. Chest CT patterns were divided according 12 items mostly suggested by radiologists and histological features were divided according to necrotising granuloma, necrotising vasculitis, eosinophilic infiltration, and hemosiderin laden macrophages as defined by a pathologist.

Results: The mean age was 57.1 years (22 men). The most common clinical manifestation other than lung manifestation was renal manifestation (62.2\%), and the most common chest CT pattern was lung involvement of vasculitis (35.1\%). In MPA patients, the major histological features were hemosiderin-laden macrophages in the alveolar space and vasculitis. In GPA patients, the major histological features were necrotizing vasculitis and necrotizing granuloma, while in EGPA patients, the major histological feature was only necrotising vasculitis. The consistency rate in GPA patients was the highest (100\%), followed by that in MPA patients (66.7\%) and EGPA patients (50.0\%).

Conclusion: When lung involvement of AAV is suspected on chest $\mathrm{CT}$, lung biopsy should be recommended for the proper classification of AAV, due to the discordance rate between radiological and histological findings in MPA and EGPA patients, but not GPA patients.
\end{abstract}

Key Words: MPA, GPA, EGPA, chest CT, histology

Received: December 31, 2018 Revised: February 28, 2019

Accepted: March 5, 2019

Co-corresponding authors: Ji-Sun Song, MD, PhD, Department of Pathology, Catholic Kwandong University College of Medicine, International St. Mary's Hospi-

tal, 25 Simgok-ro 100beon-gil, Seo-gu, Incheon 22711, Korea.

Tel: 82-32-290-3784, Fax: 82-32-290-3440, E-mail: jsong@ish.ac.kr and

Sang-Won Lee, MD, PhD, Division of Rheumatology, Department of Internal Medicine, Yonsei University College of Medicine, 50-1 Yonsei-ro, Seodaemun-gu, Seoul 03722, Korea.

Tel: 82-2-2228-1987, Fax: 82-2-393-6884, E-mail: sangwonlee@yuhs.ac

-The authors have no potential conflicts of interest to disclose.

(C) Copyright: Yonsei University College of Medicine 2019

This is an Open Access article distributed under the terms of the Creative Commons Attribution Non-Commercial License (https://creativecommons.org/licenses/ by-nc/4.0) which permits unrestricted non-commercial use, distribution, and reproduction in any medium, provided the original work is properly cited.

\section{INTRODUCTION}

Antineutrophil cytoplasmic antibody (ANCA)-associated vasculitis (AAV) is a group of vasculitides mainly affecting small vessels from capillaries to intraparenchymal arteries. AAV consists of three variants including microscopic polyangiitis (MPA), granulomatosis with polyangiitis (GPA), and eosinophilic granulomatosis with polyangiitis (EGPA). ${ }^{1} \mathrm{AAV}$ can affect various organs, including the kidneys, lungs, and peripheral nerves. Among these, lung (chest) manifestations are described as wheeze, nodules or cavities, pleurisy, infiltrate, endobronchial involvement, alveolar haemorrhage, and respiratory failure. ${ }^{2,3}$ In patients with $\mathrm{AAV}$, the rate of lung involvement ranges from $20 \%$ to $80 \%$ according to AAV variants: $60-80 \%$ for MPA, $20-$ 
$60 \%$ for GPA, 34-56\% for ANCA-positive EGPA and 60-76\% for ANCA-negative EGPA. ${ }^{4}$ The major pulmonary symptoms related to AAV are progressive dyspnoea (50-73\%) and cough (21-60\%), and are accompanied by constitutional and extrapulmonary symptoms at a rate of approximately $80 \% .{ }^{5}$ In addition, previous studies have indicated that lung involvement has an unfavourable impact on long-term prognosis and relapse rate in AAV patients. ${ }^{6-8}$

Chest computed tomography (CT) patterns of lung parenchymal lesions related to AAV include ground glass opacities, reticular shadowing, interlobular septal thickening, consolidation and honeycomb appearance. Of these findings, the most common chest CT pattern is unusual interstitial pneumonia (UIP), followed by non-specific interstitial pneumonia (NSIP). ${ }^{5,6}$ The histological features on surgical lung biopsy depend on the subtype of AAV. The typical pathologic findings in GPA are necrotizing granuloma vasculitis involving adventitia, media of small vessels; lung biopsies from EGPA patients display eosinophilic vasculitis or eosinophilic granuloma; and the histological findings in MPA are typically pulmonary haemorrhage, hemosiderin-laden macrophages in the alveolar space and neutrophilic capillaritis. ${ }^{7}$

When lung parenchymal lesions are suspected on chest Xray (CXR) or chest CT, a surgical lung biopsy is a standard test to confirm lung involvement of AAV. However, lung biopsy is an invasive and risky procedure, such that it cannot be recommended in all patients suspected of lung involvement of AAV. Thus, in clinical settings, biopsies targeting other organs such as the kidneys, nerves and maxillary sinus are performed before lung biopsy to diagnose AAV. For these reasons, previous studies have been unable to clearly answer questions on whether histological features of lung involvement are AAV variantspecific and how different the rates of consistency between radiological and histological features are among MPA, GPA and EGPA. Furthermore, to the best of our knowledge, there has been no report on the consistency between radiological and histological features in AAV patients in Korea. Hence, in this study, we investigated the chest CT patterns of $74 \mathrm{AAV}$ patients and analysed both histological features of lung involvement and the rate of consistency between radiological and histological features among MPA, GPA, and EGPA in 28 AAV patients who underwent lung biopsy within 4 weeks before and after diagnosis of AAV.

\section{MATERIALS AND METHODS}

\section{Patients}

We retrospectively reviewed the medical records of 145 patients with AAV (83 MPA, 31 GPA, and 31 EGPA patients) who had CXR results at diagnosis, according to the following inclusion criteria: 1) patients who were first classified as having AAV from October 2000 to May 2017 at the Division of Rheumatol- ogy, Department of Internal Medicine, Yonsei University College of Medicine, Severance Hospital; 2) patients with MPA who fulfilled the algorithm suggested by the European Medicines Agency (EMA) in 2007 (the 2007 EMA algorithm) and the Chapel Hill Consensus Conference (CHCC) Nomenclature of Vasculitides proposed in 2012 (the 2012 CHCC definitions), ${ }^{1,9}$ 3) patients with GPA and EGPA who met the 1990 American College of Rheumatology (ACR) classification criteria for GPA and EGPA, the 2007 EMA algorithm and the 2012 CHCC definitions. ${ }^{1,9-11}$ Also, we only included patients who had positive results of ANCA at diagnosis and had well-summarised medical records at the time of lung evaluation for assessing organbased manifestations based on Birmingham Vasculitis Activity Score (BVAS). ${ }^{2,3}$ Among 145 patients with AAV who had CXR at diagnosis, we enrolled 74 patients who underwent chest CT due to abnormalities on CXR. Of these, 28 underwent a surgical lung biopsy with concerns for lung involvement of AAV on chest CT. We reviewed the histological features of AAV and investigated consistency with histological features expected by the CT pattern in 28 of $74 \mathrm{AAV}$ patients, who had results of lung biopsy (Fig. 1). This study was approved by the Institutional Review Board of Severance Hospital (4-2017-0673).

\section{Clinical and laboratory data}

We obtained age and gender as demographic data. Perinuclear (P)-ANCA and cytoplasmic (C)-ANCA were evaluated by immunofluorescence assay. Myeloperoxidase (MPO)-ANCA and proteinase 3 (PR3)-ANCA were measured by enzyme-linked immunosorbent assay (ELISA) kits for anti-PR3 and anti-MPO (Inova Diagnostics, San Diego, CA, USA) before 2013, and by the

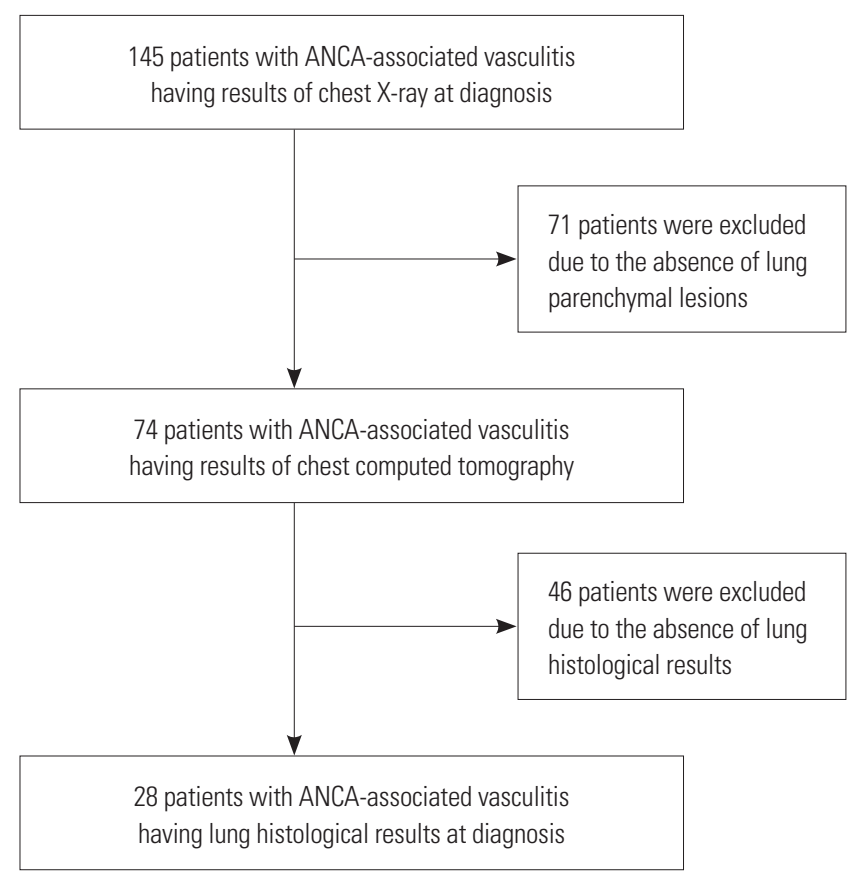

Fig. 1. Selection of the study population. ANCA, antineutrophil cytoplasmic antibody. 
novel anchor coated highly sensitive (hs) Phadia ELiA (Thermo Fisher Scientific/Phadia, Freiburg, Germany) using human native antigens, performed on a Phadia250 analyser after 2013. We reviewed the coexistence of autoimmune diseases, which can present lung parenchymal lesions similar to those of AAV. We calculated the BVAS and assessed organ-based clinical manifestations described in the BVAS. We also collected results of pulmonary function test including forced volume vital capacity (FVC), forced expiratory volume in 1 second (FEV1) and diffusing capacity of the lung for carbon monoxide (DLCO).

\section{Chest CT patterns and histological features}

Among 74 patients who underwent chest CT, 28 patients received a lung biopsy within 1 month before and after diagnosis of AAV. We divided chest CT patterns into 12 items which were mostly suggested by radiologists and were assessed as the frequency of each item: lung involvement of vasculitis (vasculitis); interstitial lung disease (ILD), including UIP, NSIP, and other ILD (not UIP or NSIP); pneumonia including bacterial or viral, tuberculosis or non-tuberculous mycobacterial and fungal pneumonia; post-inflammatory change; diffuse alveolar haemorrhage (DAH); resolved DAH; lung cancer and unclassified lung parenchymal lesions. We divided histological features compatible with AAV into 4 items as determined by a pathologist: necrotizing granuloma, necrotizing vasculitis, eosinophilic infiltration and hemosiderin-laden macrophage. We then evaluated the rate of consistency between radiological and histological features among the AAV variants. We also collected the pathologic results of other organs affected by AAV to confirm the vasculitis.

\section{Statistical analyses}

All statistical analyses were conducted using SPSS software (version 23 for Windows; IBM Corp., Armonk, NY, USA). Continuous variables are expressed as a mean \pm standard deviation, and categorical variables are reported as a number and percentage.

\section{RESULTS}

\section{Baseline characteristics of 74 patients with AAV patients with chest $C T$ results suggesting lung parenchymal lesions}

The baseline characteristics are described in Table 1. The mean age was 57.1 years and 22 patients were male. The $74 \mathrm{AAV}$ patients presented with $44 \mathrm{MPA}, 15$ GPA and 15 EGPA in this study. Fifty-four patients (73.0\%) had MPO-ANCA (or P-ANCA) and 7 (9.5\%) had PR3-ANCA (or C-ANCA). Four patients (5.4\%) had MPO-ANCA (or P-ANCA) as well as PR3-ANCA (or C-ANCA). Eight patients had concurrent autoimmune diseases that can provoke lung parenchymal lesions similar to those of AAV: 3 patients had Sjogren syndrome, 2 had systemic scle-
Table 1. Baseline Characteristics of 74 Patients with AAV and with Chest CT Results Suggesting Lung Parenchymal Lesions at Diagnosis

\begin{tabular}{|c|c|}
\hline Variables & Values \\
\hline \multicolumn{2}{|l|}{ Demographic data } \\
\hline Age (yr) & $57.1 \pm 14.2$ \\
\hline Male gender & $22(29.7)$ \\
\hline \multicolumn{2}{|l|}{ Variants of AAV } \\
\hline MPA & $44(59.5)$ \\
\hline GPA & $15(20.3)$ \\
\hline EGPA & $15(20.3)$ \\
\hline \multicolumn{2}{|l|}{ ANCA } \\
\hline MPO-ANCA (or P-ANCA) & $54(73.0)$ \\
\hline PR3-ANCA (or C-ANCA) & $7(9.5)$ \\
\hline MPO-ANCA (or P-ANCA) and PR3-ANCA (or C-ANCA) & $4(5.4)$ \\
\hline ANCA negative & $9(12.1)$ \\
\hline \multicolumn{2}{|l|}{ Autoimmune diseases other than AAV } \\
\hline Sjogren syndrome & $3(2.7)$ \\
\hline Systemic sclerosis & $2(2.7)$ \\
\hline Systemic lupus erythematosus & $1(1.4)$ \\
\hline Mixed connective tissue disease & $1(1.4)$ \\
\hline Rheumatoid arthritis & $1(1.4)$ \\
\hline \multicolumn{2}{|l|}{ Clinical manifestations other than lung involvement } \\
\hline Renal manifestation & $46(62.2)$ \\
\hline General manifestation & $42(56.8)$ \\
\hline Ear nose throat manifestation & $24(32.4)$ \\
\hline Cardiovascular manifestation & $24(32.4)$ \\
\hline Nervous systemic manifestation & $22(29.7)$ \\
\hline Cutaneous manifestation & $15(20.3)$ \\
\hline Mucous membranes/Eyes manifestation & $4(5.4)$ \\
\hline Gastrointestinal manifestation & $4(5.4)$ \\
\hline BVAS or BVAS for GPA & $13.3 \pm 7.5$ \\
\hline \multicolumn{2}{|l|}{ Pulmonary function test } \\
\hline $\operatorname{FVC}(\%)(n=42)$ & $83.6 \pm 19.5$ \\
\hline FEV1 (\%) $(n=42)$ & $87.1 \pm 24.9$ \\
\hline $\operatorname{DLCO}(\%)(n=28)$ & $81.9 \pm 21.4$ \\
\hline \multicolumn{2}{|l|}{ Major findings on chest CT } \\
\hline Reticular shadow & $13(17.6)$ \\
\hline Honeycomb & $15(20.3)$ \\
\hline Nodule & $11(14.9)$ \\
\hline Cavity & $2(2.7)$ \\
\hline Ground glass opacity & $21(28.4)$ \\
\hline Consolidation & $10(13.5)$ \\
\hline Unspecified & $2(2.7)$ \\
\hline \multicolumn{2}{|l|}{ Chest CT patterns mostly suggested } \\
\hline Lung involvement of vasculitis (vasculitis) & $26(35.1)$ \\
\hline ILD & $24(32.4)$ \\
\hline UIP & $13(17.6)$ \\
\hline NSIP & $6(8.1)$ \\
\hline Others & $5(6.8)$ \\
\hline Pneumonia & $13(17.6)$ \\
\hline Bacterial or viral & $9(12.2)$ \\
\hline
\end{tabular}


Table 1. Baseline Characteristics of 74 Patients with AAV and with Chest CT Results Suggesting Lung Parenchymal Lesions at Diagnosis (Continued)

\begin{tabular}{|cc}
\hline \multicolumn{1}{c}{ Variables } & Values \\
\hline Tuberculosis or non-tuberculous mycobacterial & $3(4.1)$ \\
\hline Fungal & $1(1.4)$ \\
\hline Post-inflammatory change & $4(5.4)$ \\
\hline Diffuse alveolar haemorrhage & $2(2.7)$ \\
\hline Resolved diffuse alveolar haemorrhage & $2(2.7)$ \\
\hline Unclassified lung parenchymal lesion & $2(2.7)$ \\
\hline Lung cancer & $1(1.4)$ \\
Reasons for no performance of lung biopsy (n=46) & \\
\hline Other vasculitis-confirming biopsy & $27(58.7)$ \\
\hline Kidney & $18(39.1)$ \\
\hline Nerve & $6(13.0)$ \\
\hline Nasal cavity & $2(4.3)$ \\
\hline Skin & $1(2.2)$ \\
\hline Refusal & $9(19.6)$ \\
\hline Pneumonia & $5(10.9)$ \\
\hline Death during the same period of hospitalisation as chest CT & $5(10.9)$ \\
\hline performance & \\
\hline AAVA
\end{tabular}

AAV, ANCA-associated vasculitis; CT, computed tomography; MPA, microscopic polyangiitis; GPA, granulomatosis with polyangiitis; EGPA, eosinophilic granulomatosis with polyangiitis; MPO, myeloperoxidase; ANCA, antineutrophil cytoplasmic antibody; P-ANCA, perinuclear ANCA; PR3, proteinase 3; C-ANCA, cytoplasmic ANCA; BVAS, Birmingham Vasculitis Activity Score; FVC, forced volume vital capacity; FEV1, forced expiratory volume in 1 second; DLCO, diffusing capacity of the lung for carbon monoxide; ILD, interstitial lung disease; UIP, usual interstitial pneumonia; NSIP, non-specific interstitial pneumonia.

Values are expressed as mean \pm standard deviation or $\mathrm{n}(\%)$.

rosis, 1 had systemic lupus erythematosus, 1 had mixed connective tissue disease, and 1 had rheumatoid arthritis. The most common clinical manifestation other than lung was renal manifestation (62.2\%), followed by general (56.8\%), ENT (32.4\%), and cardiovascular (32.4\%) manifestations. The initial mean BVAS was 13.3 . Ground glass opacity (28.4\%) was the most common major finding on chest CT, followed by honeycomb (20.3\%), reticular shadow (17.6\%), and nodule (14.9\%). The most common chest CT pattern was lung involvement of vasculitis (35.1\%), followed by ILD (32.4\%) and pneumonia (17.6\%). Among ILD, UIP (17.6\%) was the most commonly observed chest CT pattern, and among pneumonia, bacterial pneumonia (12.2\%) was most frequently observed. Pulmonary function test showed that mean FVC and FEV1 were $83.6 \%$ and $87.1 \%$ and that mean DLCO was $81.9 \%$.

\section{Lung histology and consistency rate between radiological and histological features}

Forty-six of 74 patients were suspected lung involvement of AAV but did not undergo lung biopsy for the following reasons: AAV was confirmed from other organ tissues in 27 patients (58.7\%), biopsy was refused in $9(19.6 \%)$, pneumonia was expected to be aggravated after lung biopsy in 5 (10.9\%), and 5 patients expired (10.9\%). Among 14 MPA patients, 6 patients ex- hibited chest CT findings suggesting vasculitis, whereas 6 patients exhibited histological features of AAV. On histology, hemosiderin laden macrophage was observed in 3 MPA patients and necrotising vasculitis was noted in 3 MPA patients. Only 4 of 6 patients with histological evidence of AAV had chest CT findings suggesting vasculitis. Thus, the consistency rate between radiological and lung histological findings was $66.7 \%$ in MPA patients. All 9 GPA patients exhibited both chest CT and lung histological findings of AAV. On histology, necrotising granuloma, necrotising vasculitis and eosinophilic infiltration were observed in 6, 9, and 1 GPA patients, respectively. Thus, the consistency rate between radiological and lung histological findings was $100 \%$ in GPA patients. Meanwhile, among 5 EGPA patients, 2 patients exhibited necrotising vasculitis on histology and furthermore 1 of 2 patients had chest CT findings suggesting vasculitis. Thus, the consistency rate between radiological and lung histological findings was $50.0 \%$ in EGPA patients (Table 2).

\section{DISCUSSION}

In this study, we investigated chest CT patterns in $74 \mathrm{AAV}$ patients and analysed both the histological features of lung involvement and the consistency between radiological and histological results in $28 \mathrm{AAV}$ patients who underwent lung biopsy according to AAV variants. Of 145 patients who had results of CXR at diagnosis of AAV, 74 patients (51.0\%) underwent chest CT. The most frequent extra-pulmonary manifestation of them was renal manifestation, followed by general manifestation. Similarly, previous reports revealed that patients with AAV exhibited kidney-related symptoms in up to $100 \%$ and constitutional symptoms in approximately $80 \% .^{5}$

In this study, we founded that lung histological features differ among AAV variants. In MPA patients, the major histological features were necrotizing vasculitis and hemosiderin-laden macrophages in MPO-ANCA positive patients, and none exhibited necrotising granuloma or eosinophilic infiltration in lung tissues. Meanwhile, in GPA patients, the major histological features were necrotizing vasculitis and necrotizing granuloma, and the major histological feature of EGPA patients was only necrotizing vasculitis.

The consistency rates between radiological and lung histological findings were based on AAV variants. The consistency rate in GPA patients was the highest (100\%), followed by that in MPA patients (66.7\%) and EGPA patients (50.0\%). Two patients suspected of vasculitis on chest CT had no evidence of AAV on lung histology but they were finally classified as MPA by renal histological findings. Interestingly, 1 patient suspected of other ILD and 1 patients suspected of pneumonia exhibited histological features of MPA. Meanwhile, 1 of 2 EGPA patients with histological features compatible with AAV was suspected of pneumonia, rather than AAV on chest CT. Therefore, in MPA 


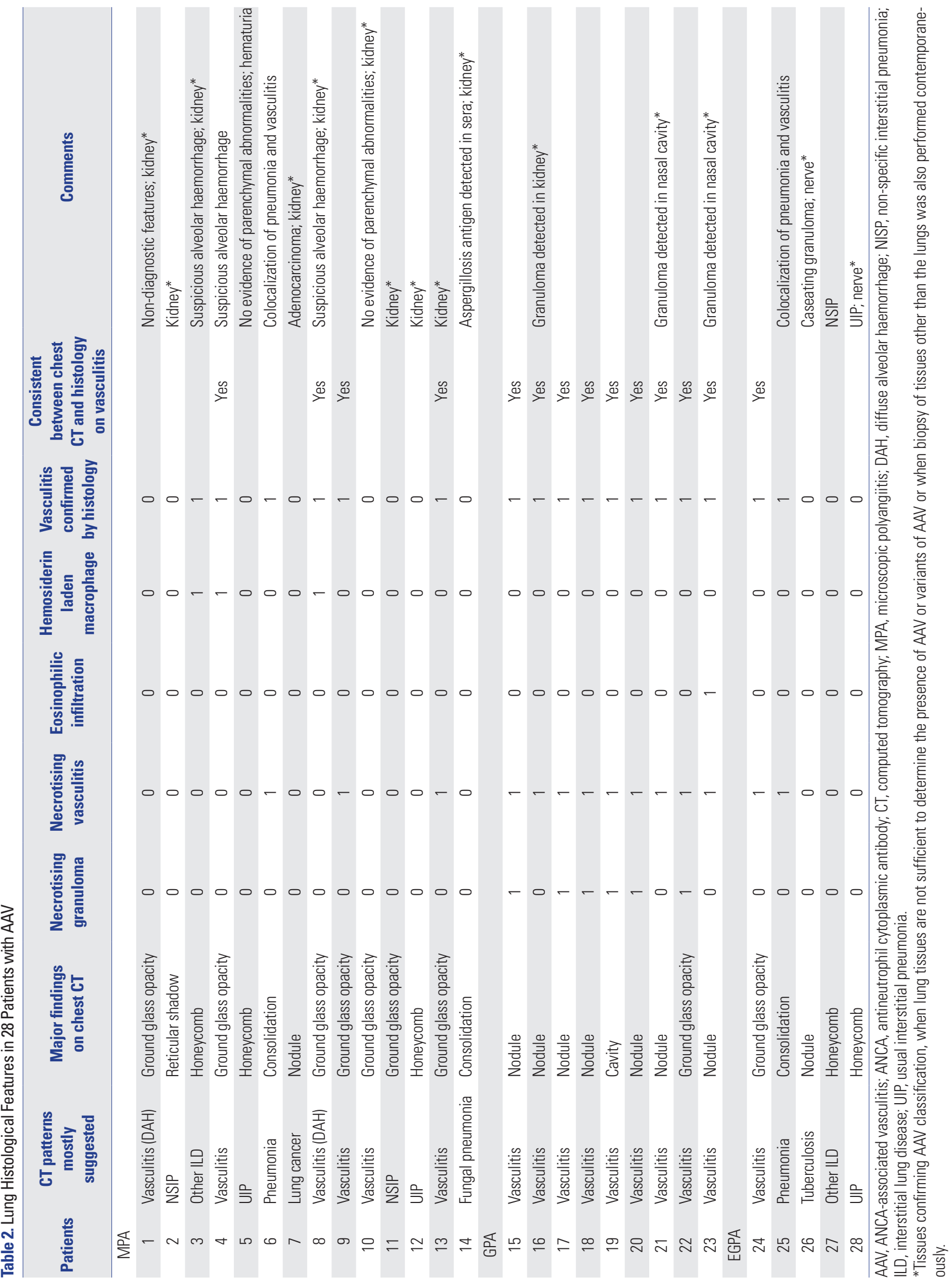


and EGPA patients, when lung involvement of AAV is suspected on chest CT, lung biopsy should be recommended for proper classification of AAV, due to discordance between radiological and histological findings.

While radiological features on chest CT could suggest GPA, physicians should not put blind belief in chest CT patterns showing cavitary lung parenchymal lesions before the final classification of AAV. It could be reasonably speculated that lung cavitary parenchymal lesions are important predictive clues for GPA. However, one patient with MPA who exhibited cavitary parenchymal lesions in chest CT was finally confirmed to have a fungal infection on lung biopsy. In this case, MPA was confirmed by MPO-ANCA positivity and renal biopsy. Furthermore, the aspergillosis antigen was detected in the serum of this patient and typical Aspergillus hyphae was observed on lung histology. ${ }^{12,13}$ The halo sign in chest CT images has been used to discern the aetiologies of pulmonary cavitary nodules; however, this can be observed in various diseases beyond GPA, including invasive aspergilloma. ${ }^{14}$ Herein, three of 9 GPA patients who exhibited cavitary lesions in chest CT underwent lung biopsy to exclude other lung pathologic conditions including fungal infection despite the presence of histology of nasal cavity compatible with GPA in our study. ${ }^{15}$

One MPA patient and one EGPA patient had overlapping syndromes with AAV and Sjogren syndrome. Both patients had anti-SSA/Ro antibody and decreased lacrimal and salivary gland function. In these patients, chest CT mostly suggested UIP, and lung biopsies revealed patchy interstitial fibrosis with honeycomb change, which was consistent with a UIP pattern, with many lymphoid follicles. ${ }^{16,17}$ When AAV was accompanied by autoimmune connective tissue disease, at least confined to this study, lung histology did not seem helpful to confirm vasculitis. Also, lung biopsy alone could not confirm AAV when chest CT showed diffuse ground glass opacity lesions suggestive of ILD, pulmonary haemorrhage, and atypical pneumonia in patients with AVV. Meanwhile, the diagnosis of MPA was usually made through kidney biopsy, and that of EGPA was done through nerve biopsy. However, physicians should consider a lung biopsy, because other aetiologies, such as fungal infection, tuberculosis and lung cancer, must be excluded before treatment for AAV.

The administration of glucocorticoid before lung biopsy may have an influence on histological features of lung parenchyma and may confuse the precise classification of AAV. Therefore, we reviewed the medical records of $28 \mathrm{AAV}$ patients who underwent lung biopsy and counted the number of patients who received glucocorticoids before lung biopsy. Glucocorticoids were administered to five MPA patients before lung biopsy. Two of the five MPA patients had previously received glucocorticoids due to DAH. Four of the five patients exhibited histological features compatible with MPA, while one patient did not. Thus, we assume that the administration of glucocorticoid might hide the histological features of AAV in MPA patients who exhibit ground-glass opacity on chest CT, despite the small number of patients and the retrospective study design.

Our study has merit in that we, for the first time, described both histological and radiological features in the same AAV patients, compared them and assessed the consistency between them in all variants of AAV. However, our study also has several limitations: First, we retrospectively reviewed medical records of AAV patients, which prohibited us from interpreting radiological features with more information, particularly clinical symptoms and signs at diagnosis. Second, only 28 of 74 patients who exhibited abnormal lung parenchymal findings on chest imaging studies underwent lung biopsy because of various clinical reasons. Accordingly, we believe that additional cases of lung biopsy could have further clarified the link between radiological and histological information in AAV patients.

In conclusion, the consistency rate in GPA patients was the highest (100\%), followed by that in MPA patients $(66.7 \%)$ and EGPA patients (50.0\%). Therefore, in MPA and EGPA patients, when lung involvement of AAV is suspected on chest CT, lung biopsy should be recommended for the proper classification of AAV, due to discordance between radiological and histological findings.

\section{ACKNOWLEDGEMENTS}

This study was supported by a faculty research grant from Yonsei University College of Medicine (6-2016-0145).

\section{AUTHOR CONTRIBUTIONS}

Conceptualization: Song JS, Lee SW. Data curation: Park HJ, Song JS, Lee SW. Formal analysis: Park HJ, Jung SM, Song JS, Lee SW. Funding acquisition: Park YB, Lee SW. Investigation: Park HJ, Jung SM, Song JS, Lee SW. Methodology: Song JJ, Song JS, Lee SW. Project administration: Park HJ, Song JS, Lee SW. Resources: Park HJ, Song JS, Lee SW. Software: Park HJ, Song JS, Lee SW. Supervision: Song JS, Lee SW. Validation: Jung SM, Song JJ, Park YB. Visualization: Park HJ, Song JS, Lee SW. Writing—original draft: Park HJ. Writing—review \& editing: Park HJ, Song JS, Lee SW.

\section{ORCID iDs}

$\begin{array}{ll}\text { Hee-Jin Park } & \text { https://orcid.org/0000-0002-6324-5262 } \\ \text { Seung Min Jung } & \text { https://orcid.org/0000-0003-3465-2181 } \\ \text { Jason Jungsik Song } & \text { https://orcid.org/0000-0003-0662-7704 } \\ \text { Yong-Beom Park } & \text { https://orcid.org/0000-0003-4695-8620 } \\ \text { Ji-Sun Song } & \text { https://orcid.org/0000-0003-4877-3128 } \\ \text { Sang-Won Lee } & \text { https://orcid.org/0000-0002-8038-3341 }\end{array}$

\section{REFERENCES}

1. Jennette JC, Falk RJ, Bacon PA, Basu N, Cid MC, Ferrario F, et al. 2012 revised International Chapel Hill Consensus Conference Nomenclature of Vasculitides. Arthritis Rheum 2013;65:1-11.

2. Mukhtyar C, Lee R, Brown D, Carruthers D, Dasgupta B, Dubey S, 
et al. Modification and validation of the Birmingham Vasculitis Activity Score (version 3). Ann Rheum Dis 2009;68:1827-32.

3. Stone JH, Hoffman GS, Merkel PA, Min YI, Uhlfelder ML, Hellmann $\mathrm{DB}$, et al. A disease-specific activity index for Wegener's granulomatosis: modification of the Birmingham Vasculitis Activity Score. International Network for the Study of the Systemic Vasculitides (INSSYS). Arthritis Rheum 2001;44:912-20.

4. Millet A, Pederzoli-Ribeil M, Guillevin L, Witko-Sarsat V, Mouthon L. Antineutrophil cytoplasmic antibody-associated vasculitides: is it time to split up the group? Ann Rheum Dis 2013;72:1273-9.

5. Alba MA, Flores-Suárez LF, Henderson AG, Xiao H, Hu P, Nachman $\mathrm{PH}$, et al. Interstital lung disease in ANCA vasculitis. Autoimmun Rev 2017;16:722-9.

6. Hervier B, Pagnoux C, Agard C, Haroche J, Amoura Z, Guillevin L, et al; French Vasculitis Study Group. Pulmonary fibrosis associated with ANCA-positive vasculitides. Retrospective study of 12 cases and review of the literature. Ann Rheum Dis 2009;68:404-7.

7. Travis WD, Leslie KO, Beasley MB. Pulmonary vasculitis and pulmonary hemorrhage. In: Leslie KO, Wick MR. Practical pulmonary pathology: a diagnostic approach. Philadelphia (PA): Elsevier;2018. p.365-400.

8. Tzelepis GE, Kokosi M, Tzioufas A, Toya SP, Boki KA, Zormpala A, et al. Prevalence and outcome of pulmonary fibrosis in microscopic polyangiitis. Eur Respir J 2010;36:116-21.

9. Watts R, Lane S, Hanslik T, Hauser T, Hellmich B, Koldingsnes W, et al. Development and validation of a consensus methodology for the classification of the ANCA-associated vasculitides and polyarteritis nodosa for epidemiological studies. Ann Rheum Dis 2007; 66:222-7.
10. Leavitt RY, Fauci AS, Bloch DA, Michel BA, Hunder GG, Arend WP, et al. The American College of Rheumatology 1990 criteria for the classification of Wegener's granulomatosis. Arthritis Rheum 1990;33:1101-7.

11. Masi AT, Hunder GG, Lie JT, Michel BA, Bloch DA, Arend WP, et al. The American College of Rheumatology 1990 criteria for the classification of Churg-Strauss syndrome (allergic granulomatosis and angiitis). Arthritis Rheum 1990;33:1094-100.

12. Shin B, Koh WJ, Jeong BH, Yoo H, Park HY, Suh GY, et al. Serum galactomannan antigen test for the diagnosis of chronic pulmonary aspergillosis. J Infect 2014;68:494-9.

13. Muldoon EG, Sharman A, Page I, Bishop P, Denning DW. Aspergillus nodules; another presentation of Chronic Pulmonary Aspergillosis. BMC Pulm Med 2016;16:123.

14. Godoy MC, Viswanathan C, Marchiori E, Truong MT, Benveniste MF, Rossi S, et al. The reversed halo sign: update and differential diagnosis. Br J Radiol 2012;85:1226-35.

15. Shimon G, Yonit WW, Gabriel I, Naama BR, Nissim A. The "Treein-Bud" pattern on chest CT: radiologic and microbiologic correlation. Lung 2015;193:823-9.

16. Egashira R, Kondo T, Hirai T, Kamochi N, Yakushiji M, Yamasaki F, et al. CT findings of thoracic manifestations of primary Sjögren syndrome: radiologic-pathologic correlation. Radiographics 2013; 33:1933-49.

17. Watanabe M, Naniwa T, Hara M, Arakawa T, Maeda T. Pulmonary manifestations in Sjogren's syndrome: correlation analysis between chest computed tomographic findings and clinical subsets with poor prognosis in 80 patients. J Rheumatol 2010;37:365-73. 